\title{
A Context-Aware Application to Increase Elderly Users Compliance with Physical Rehabilitation Exercises at Home via Animatronic Biofeedback
}

\author{
Borja Gamecho · Hugo Silva · José Guerreiro · Luis Gardeazabal · Julio Abascal
}

Received: date / Accepted: date

\begin{abstract}
Biofeedback from physical rehabilitation exercises has proved to lead to faster recovery, better outcomes, and increased patient motivation. In addition, it allows the physical rehabilitation processes carried out at the clinic to be complemented with exercises performed at home. However, currently existing approaches rely mostly on audio and visual reinforcement cues, usually presented to the user on a computer screen or a mobile phone interface. Some users, such as elderly people, can experience difficulties to use and understand these interfaces, leading to non-compliance with the rehabilitation exercises. To overcome this barrier, latest biosignal technologies can be used to enhance the efficacy of the biofeedback, decreasing the complexity of the user interface. In this paper we propose and validate a contextaware framework for the use of animatronic biofeedback, as a way of potentially increasing the compliance of elderly users with physical rehabilitation exercises performed at home. in the scope of our work, animatronic biofeedback entails the use of pre-programmed actions on a robot that are triggered in response to certain changes detected in the users' biomechanical or electrophysiological signals. We use electromyographic and accelerometer signals, collected in real time, to monitor the performance of the user while execut-
\end{abstract}

B. Gamecho(西) · L. Gardeazabal · J. Abascal

UPV-EHU University of the Basque Country

Paseo Manuel Lardizabal, 1,

20018, Donostia-San Sebastián

Tel.: +034-943-015113

Fax: +034-943-015590

E-mail: borja.gamecho@ehu.eus

H. Silva

IT - Instituto de Telecomunicações

IST - Universidade de Lisboa, Lisboa, Portugal

J. Guerreiro

Strathclyde University, Glasgow, Scotland ing the exercises, and a mobile robot to provide animatronic reinforcement cues associated with their correct or incorrect execution. A context-aware application running on a smartphone aggregates the sensor data and controls the animatronic feedback. The acceptability of the animatronic biofeedback has been tested on a set of volunteer elderly users. The results suggest that the participants found the animatronic feedback engaging and of added value.

Keywords Context-Awareness, Mobile Computing, Physiological Signals, Human-Computer Interaction.

\section{Introduction}

Every year a vast number of people undergo physical rehabilitation due to work related injuries [1], disability [2], and other conditions. Within the portfolio of tools that therapists currently have at their disposal, biofeedback has become particularly popular $[3,4]$, with clinical evidence showing that it is an engaging technique with multiple benefits for the patient [3-6]. As a way of improving the effectiveness of treatments, one of the latest trends has been the extension of the rehabilitation process to people's homes with biofeedback exercises designated by the therapist to be performed autonomously by the patient at home, in-between sessions at the clinic [7-9].

A major advantage of biofeedback is the possibility of deriving objective performance indicators from direct physiological measurements in real time, and using them for live progress monitoring and guidance of the rehabilitation exercises to maximize the potential outcomes. A critical aspect in any biofeedback system are positive and negative reinforcement cues, provided to the patient in real time when a given exercise is being executed. These are used as a way of confirming whether the goals set by the therapist are being met or not. Reinforcement cues used in biofeedback are 
generally visual, acoustic, and/or haptic signals delivered through a computer or mobile device, acting as the interface with the patient. However, aspects such as screen size, interfaces, lack of technological proficiency, reduced sight or hearing abilities, and other factors associated with the more traditional feedback methods can pose difficulties to specific user groups (such as elderly patients), ultimately leading to poor compliance in home exercises.

In this paper we propose a new approach based on a context-aware application, which translates context information (gathered from physiological sensor data acquired in real time) into animatronic feedback with underlying semantics (see Fig. 1). Given that it is mostly based on physical rather than digital interfaces, our approach makes way for a more natural interaction and relaxes several constraints found in other approaches. We believe that this context-aware animatronic biofeedback perspective can greatly contribute to further enhance the set of tools available for therapists, especially as far as methods for increasing compliance with home exercises in elderly patients are concerned.

\section{Related Work}

As recently argued by Chandra, Oakley and Silva [10], physical rehabilitation at home using biofeedback has multiple potential drawbacks, user engagement being the most important. Positive and/or negative reinforcement cues provided to the user during the exercises play a major role in user engagement. In this section we present a summary of approaches found in the state-of-the-art supporting physical rehabilitation at home using biofeedback.

\subsection{Audiovisual Biofeedback}

Audiovisual feedback is the most widespread approach ever since biofeedback started to being used, focuses on the use of either visual or acoustic cues (or a mixture of both) for positive and/or negative reinforcement. Liu and Quian [11] proposed a system for stroke rehabilitation that uses cameras to monitor the user's motion and delivers visual feedback, by means of a projector, to cue the user. This system is fairly complex and costly for users to setup and use autonomously.

Also based on motion tracking, the work by Lange et al. [12] proposes a gamification approach to physical rehabilitation, in which a low cost depth sensing camera is used together with a game of balance training for adults suffering from neurological injury. Given that cameras can impose constraints regarding the patient's relative position to the camera, Daponte, de Vito, and Sementa [13] proposed a system based on wearable Inertial Measurements Units (IMUs) for range of limb motion measurement in home rehabilitation.
The main drawback of motion data -regardless whether it is collected by camera or IMU- is that it only provides an indirect measurement of the underlying physiological processes associated with a given exercise, while other signals such as Electromyography (EMG) provide much finer and more direct measurements. Aung and Al-Jumaily [14] present a shoulder rehabilitation system that aims to increase the motivation and autonomous effort of users while doing physical rehabilitation exercises. Their system uses a number of real time line graphics and other visual cues triggered in response to the muscle activity as measured by EMG sensors. The user can choose from a set of four game-like interaction activities that provide feedback cues on a computer screen.

As a way of simplifying the overall setup and complexity of the procedures involved in EMG biofeedback, Farjadian, Sivak and Mavroidis, [15] proposed a t-shirt that acquires multiple EMG channels and heart rate data. A smartphone interface provides audiovisual feedback, enabling the user to observe and adjust the exercises in real time. The work by Daponte et al. [16] also evaluates the use of EMG data in post-stroke rehabilitation, combining it with range of motion data acquired using a goniometer.

Over the years a number of commercial devices available for home rehabilitation have also been made available. Thought Technology[17], MindMedia[18], and PLUX[19] all provide a range of devices for real time audiovisual feedback, which users can follow while doing physical rehabilitation exercises.

\subsection{Kinesthetic Biofeedback}

Although audiovisual feedback is useful, in some cases it is advantageous for users to have a mechanical or haptic feedback either as an alternative or as a complement. For this reason, abundant research has been devoted to kinesthetic biofeedback techniques.

A biomechatronic device named RUPERT has been presented by Sugar et al. [20], as a way to provide an easy-touse assistance to enable the patient and therapist to achieve more systematic therapy at home or at the clinic. This device has been designed as a wearable robot for upper extremity physical rehabilitation using biofeedback in stroke patients. In [21], Lunenburger et al. combine a virtual environment with a robot-assisted gait training unit, in an attempt to increase the motivation to perform the exercise.

Physical rehabilitation exercises may be required for a number of body regions and patient conditions. For instance, Monaco et al. [22] proposed a neuro-rehabilitative platform for bedridden post-stroke patients. This platform enables active and passive control of the motion in a range of motor tasks. In [23], the authors have used real time sensing and haptic feedback to train tibia and trunk angle changes during gait. 

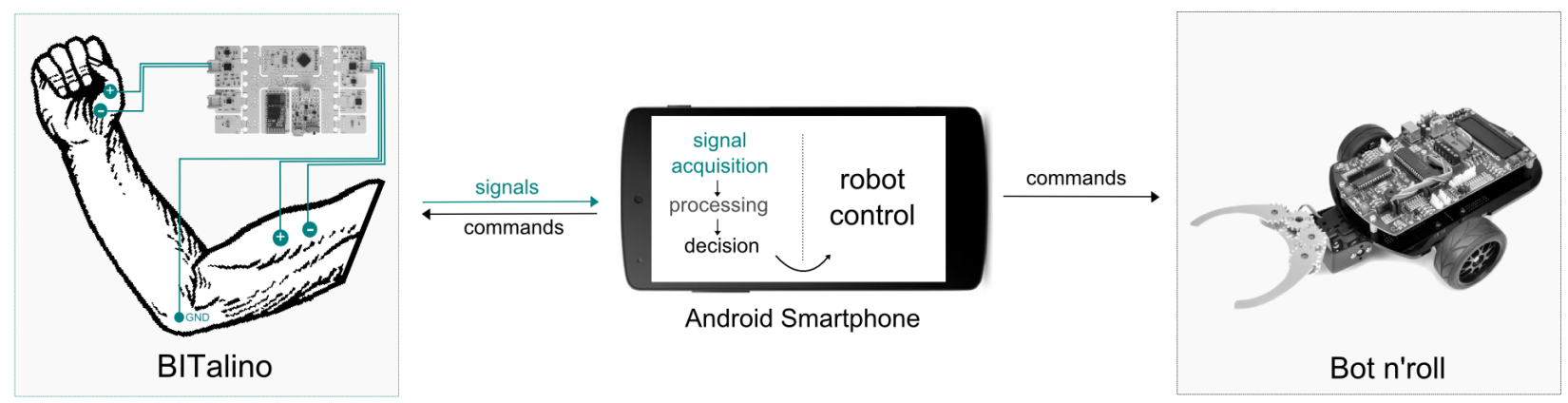

Fig. 1: Main elements of the proposed animatronic biofeedback system

Electromyography (EMG) has also been used together with kinesthetic feedback; Casellato et al. [24], explored the use of visual-haptic biofeedback during upper limb movements, as a way of testing if augmented sensory feedbacks can induce motor control improvement in patients with primary dystonia.

Another dimension of kinesthetic biofeedback is the delivery of feedback stimuli using electrical stimulation rather than biomechatronic or haptic feedback. An example of such a system is shown in the work by Banerji et al. [25], which used functional electrical stimulation as a tool to augment rehabilitation in post-stroke patients.

\section{Proposed Approach}

In this work we propose a low-cost, easy to deploy alternative to the above mentioned biofeedback categories. It is based on animatronic biofeedback mediated by a contextaware application, which uses multimodal physiological data as input.

\subsection{Animatronic Feedback}

Visual and auditory biofeedback has been amply studied in the literature. In this work we choose an animatronic feedback approach in order to provide positive and negative reinforcement cues. We want to test whether the actions of the robot biofeedback response would be of added value to the user, given that it is a physical rather than a audiovisual interface. This approach has the advantage of only depending on a physical interface, programmed to perform a pre-set series of actions related to the accomplishment, or not, of the goals set by the therapist.

Unlike software user interfaces displayed on a computer or mobile phone screen, which are not necessarily accessible in terms of interaction, animatronic feedback offers a more natural interaction with the reinforcement cues. Thus potentially being easier to understand and more engaging for the user. The robot represents the "persona" of a robotic pet moving in front of the user and delivering the animatronic feedback cues. This makes it particularly suitable for user groups less proficient with digital interfaces, such as the elderly.

For our work we used a Bot'n Roll mobile robot [26], which combines a set of physical actuators, namely servo wheels to allow the robot movements, and a servo claw used to grab objects. It can be controlled in real time by means of a wireless communication module based on Bluetooth technology (Class II v2.0) with a baud-rate of 38.400 bps. Using these features we programmed animatronic feedback actions that can be triggered as positive reinforcement cues when the user is correctly performing a given exercise, namely:

1. Forward or backward motion;

2. Turning left or right;

3. Closing and opening the claw;

The negative reinforcement cue is common to all exercises, and is expressed by the robot going backwards to the place it was before the last movement.

If the robot remains still without moving it could mean various things:

1. After positive feedback: the current exercise has been performed well.

2. After negative feedback: the last exercise may be tried again.

3. In the remaining cases: the current movement has not been correctly performed yet.

\subsection{Exercise Assessment}

A key element in biofeedback is the ability to measure how a given exercise is being performed. The most common exercises involve either the musculoskeletal system, biomechanical activities, or a combination of both. Electromyography 
(EMG) provides a direct measurement of the recruitment of one or several groups of muscles. Accelerometry (ACC), on the other hand, can be used for biomechanical assessment (e.g. range of motion, limb tilt, etc.). For this part of our work we used the BITalino biosignal acquisition platform [27].

BITalino combines EMG and ACC sensors with a wireless communication module that uses Bluetooth technology (Class II v2.0), providing both biosignals acquisition and connectivity to a base station (in our case a smartphone). We used the BITalino Board in a configuration that acquires EMG and ACC (Z-axis) signals (10 bits @ $f s=100 \mathrm{~Hz}$ ), streaming the raw data via Bluetooth with a baudrate of $115.200 \mathrm{bps}$ to the smartphone. Given that BITalino only outputs raw data, we had to devise a set of algorithms to convert this data into meaningful events.

\subsubsection{Muscle Contraction Detection}

For EMG data, we used an onset detection algorithm consisting of two stages, namely a processing block to filter the signal and compute the envelope of the EMG signal, and a decision rule block (see Fig. 2). The processing block uses a sliding window of $M=40$ samples, without overlapping between windows, to perform a moving average filtering of the signal as described by Equation 1 in which $x[n]$ is the input signal (EMG), $s[n]$ is the filtered signal, and $M$ is the moving average window size without overlapping. For a sampling frequency of $100 \mathrm{~Hz}$, the $-3 \mathrm{~dB}$ cut-off frequency is approximately $30 \mathrm{~Hz}$ and the gain is 2 at $0 \mathrm{~Hz}$. The phase shift is $20 \mathrm{~ms}$ (two samples):

$s[n]=\frac{1}{M} \sum_{j=0}^{M-1} x[n-j]$.

Afterwards, signal rectification is performed by subtracting the DC component $s[n]$ and computing the absolute value of each point within the sliding window, as defined by Equation 2 .

$z[n]=\left|s[n]-\frac{1}{M} \sum_{j=0}^{M-1} s[n-j]\right|$.

The processing stage finishes by computing the average value of the sliding window, as described in Equation 3 (where $M=40$ as previously described). In the end, we have one resultant value, $y[n]$, for each $M$ samples of the raw input signal. Example data for biceps contractions can be seen in Fig. 3 (a).

$y[n]=\frac{1}{M} \sum_{j=0}^{M-1} z[n-j]$.

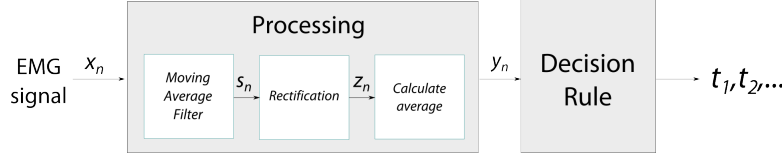

Fig. 2: Signal Processing for the EMG

Finally, a simple decision rule is applied to $y[n]$ to determine if there is a muscle contraction when $y[n]>$ threshold. The threshold value is defined as a percentage of what is called the Maximum Voluntary Contraction (MVC). In the calibration stage, the user is asked to voluntarily contract each monitored muscle in order to determine the maximum muscle activation amplitude he can produce (the MVC). The threshold is manually defined as a \% of the MVC; the fact that this is a relative value makes it insensitive to variable factors between exercising sessions (e.g. skin moisture, different electrodes, different devices, etc.).

\subsubsection{Limb Tilt and Motion Detection}

The strategy used for ACC data processing follows the same approach. However, the processing stage consists only of a low-pass filter implemented with a moving average filter, as described by Equation 1. Similarly to the EMG approach, the ACC decision rule is applied to determine the ACC value in each instant and compared it with a threshold value interval. The thresholds for the ACC were calculated by the analysis of the values for the processed data and mapping them with the limb angles. As an example of limb tilt, three different positions of an accelerometer attached to the wrist can be seen in Fig. 3 (b).

\subsection{Context-Aware Application}

We adopted an approach based on context-aware systems for the development of the mobile application. Following the definition of context given by Dey et al. [28], an application is context-aware if the interaction between the user and the application is affected by relevant information related to the entities of that context. Usually these entities are people, objects and the environment where the interaction takes place.

To facilitate the use of the context, our applications use an extended version of the MobileBIT framework [29]. MobileBIT is a framework for rapid prototyping of Android applications intended for real-time data acquisition, processing, recording, communication and visualization for the telemedicine and mobile health domains. It was adapted to deliver context information to mobile applications.

In MobileBIT applications, context information is obtained from sensors and processed into meaningful data to 

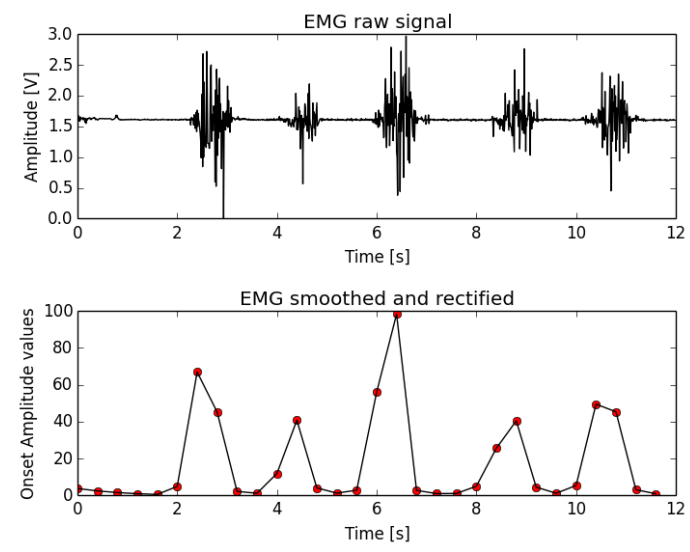

(a) EMG
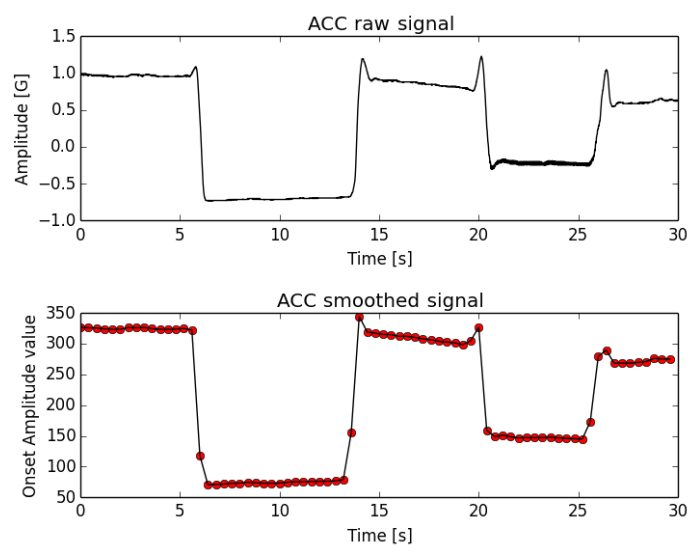

(b) ACC

Fig. 3: EMG and ACC signals were used to evaluate the adopted algorithm. The top row shows the EMG (left) and ACC (right) raw signals, and the bottom row shows the signal obtained at the final stage of the adopted algorithm. It is important to highlight how the output of the algorithm we have adopted facilitates the onset detection on the EMG signals

generate semantically complex information. MobileBIT allows developers to define relevant pieces of context information and use them to create context-aware applications.

In this case, the context information is the contraction of muscles and tilt movements of limbs following the assessment of the Section 3.2. We apply it to the right arm muscles and movements in our context-aware applications. One Android device receives the EMG and ACC data from the BITalino with a sampling rate of $100 \mathrm{~Hz}$. Every $400 \mathrm{~ms}$ (40 samples), the signals are processed to obtain a value that is compared with each threshold interval. The application is notified of every new context information acquired by the MobileBIT framework and sends a command to move the robot when a biofeedback response is required. The obtained context information can be seen in Table 1 .

From the developers perspective, mechanisms provided by MobileBIT make it possible to create context-aware mobile applications. From the end-user perspective, these applications may hide the notion of context and they are perceived as enhanced mobile applications. In the case of this work, the final application follows a natural interaction approach using arm gestures and movements as a way of interacting with the robot.

\subsubsection{The ToBITas Use Case}

On a preliminary stage, we built an application called ToBITas [30], a proof of concept for a context-aware application that controls a mobile robotic platform using physiological sensors. Users can activate different responses in the robot in real time using two EMG channels and one ACC channel of the BITalino (see Table 1 for a detailed description of the interaction).

\subsubsection{Physical Rehabilitation Use Case}

Following this, we depicted a more constrained scenario with a step by step control mode with routines and tasks comparable to a real set of rehabilitation exercises.

Two types of exercises well performed by the user will trigger different actions in the robot:

1. Activating the biceps (e.g. folding the arm) for $T$ seconds sends a command to the robot to move forward for a distance of $40 \mathrm{~cm}$.

2. Tilting the wrist to the left or right for $T$ seconds will animate the robot to turn in the same direction for 90 degrees.

In this scenario physiotherapists define the chaining of these two kinds of exercises as routines. Usually the same exercise would be repeated a number of times in the routine. If the exercise is interrupted, the robot will activate the negative biofeedback cue and move back to the initial position. Consequently, the user must perform the last exercise again. We consider the following events as interruptions:

1. For muscles: When the contraction value is below the threshold set up in the calibration phase.

2. For tilt positions: When the value of the accelerometer is outside the region defined for an exercise.

\section{Experimental Evaluation: Rehabilitation Routines with Elderly People}

We performed a usability study and assessed the value of animatronic feedback to increase user engagement. We considered this to be a mandatory first step in order to provide 
Table 1: The relationship between the acquired signals, the context information and the system behaviour

\begin{tabular}{llll}
\hline Signal & User Action & Context Information & Robot Command \\
\hline EMG_1 & The user folds his arm & Action_detected: Right_arm_folded & Move Forward \\
\hline EMG_2 & The user closes his hand & Action_detected: Hand_Closed & Open/Close the Claw \\
\hline \multirow{2}{*}{ ACC } & Tilts the wrist to the left & Position_detected: Wrist_up & Move Right \\
& $\begin{array}{l}\text { Tilts the wrist to the right } \\
\text { Wrist in central position }\end{array}$ & $\begin{array}{l}\text { Position_detected: Wrist_down } \\
\text { Position_detected: Wrist_side }\end{array}$ & $\begin{array}{l}\text { Move Left } \\
\text { Don't Move }\end{array}$
\end{tabular}

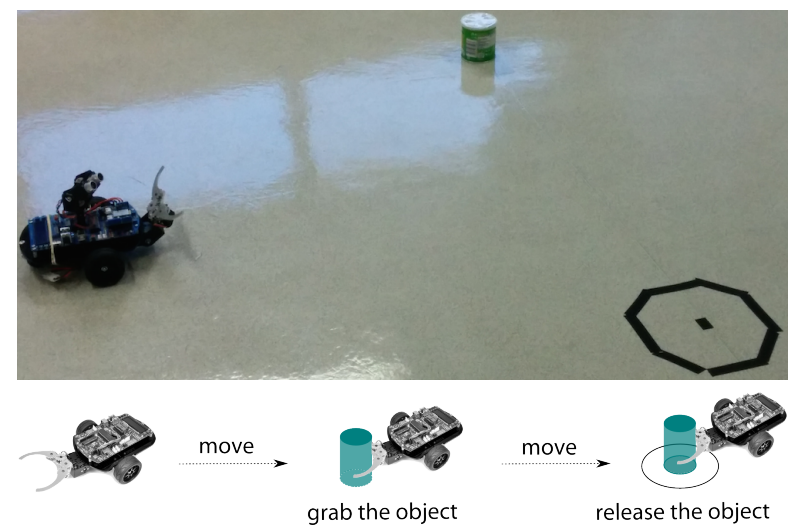

Fig. 4: Experimental setup and task description for the evaluation of ToBITas use case

therapists with evidence that could support a more comprehensive clinical study in future work. Hence, we only involved participants not enrolled in physical rehabilitation.

This evaluation was carried out to answer the following research question: Does the animatronic biofeedback have any advantages when it is used for rehabilitation exercises for elderly people? In order to answer this question, we compared visual on-screen biofeedback obtained using an Android TV application (Method A) with our approach of animatronic biofeedback (Method B) implemented by the application as described in Section 3.3.2.

\subsection{Testing the Overall Setup, ToBITas Case Study}

Before the evaluation with real users we developed a case study to validate the equipment that can be found in Gamecho et al. [30]. The objective was to try a free control mode with young subjects implemented in the application described in Section 3.3.1. The original goal was to perform a set of movements in real time (see Fig. 4 for the original routine description). Results showed that users were able to understand and quickly learn how to use our approach (see Table 2 and Fig. 5).
Table 2: Summary of the task performance results for each group of participants measured in seconds for the test experiment. (A: Novice participant, B: Participant with some experience on similar systems controlled by gestures, $\mathrm{C}$ : ToBITas developers). $T_{n}$ refers to the attempt

\begin{tabular}{llllll}
\hline Group & $T_{1}[\mathrm{~s}]$ & $T_{2}[\mathrm{~s}]$ & $T_{3}[\mathrm{~s}]$ & $\mu[\mathrm{s}]$ & $\sigma[\mathrm{s}]$ \\
\hline A (7 users) & 140 & 93 & 57 & 96 & 59 \\
B (4 users) & 32 & 45 & 48 & 42 & 7 \\
C (2 users) & 25 & 38 & 24 & 29 & 6 \\
Average & 89 & 70 & 49 & 69 & 16 \\
\hline
\end{tabular}

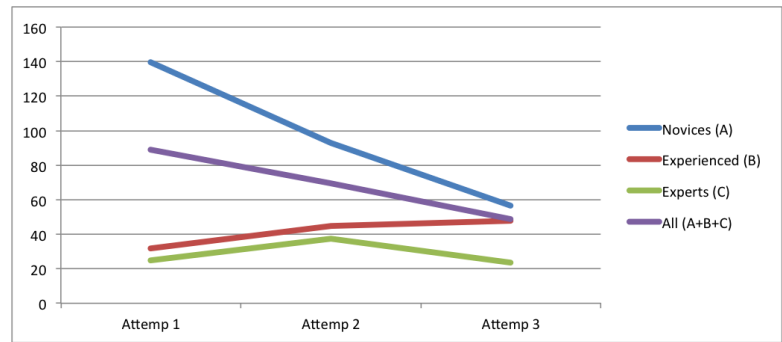

Fig. 5: Progression chart from the first evaluation of the ToBITas Case Study, the learning effect is noticeable for Novice users

\subsection{Method}

For the next step in the evaluation we redesigned the animatronic biofeedback from a continuous feedback control mode to a step by step one. In this second approach, only one movement can be activated at the same time to perform one of the exercises described in Section 3.3.2. The mobile application waits until an exercise has finished to enable the following one. In our previous experiment (see Section 4.1) we used the thenar eminence muscle, which proved not to be a good choice for the type of exercises we envisioned. Therefore we decided to eliminate it from the study. Afterwards three different exercises were considered for the evaluation: biceps contraction, wrist tilt to the left and wrist tilt to the right. The exercises were designed taking into account 


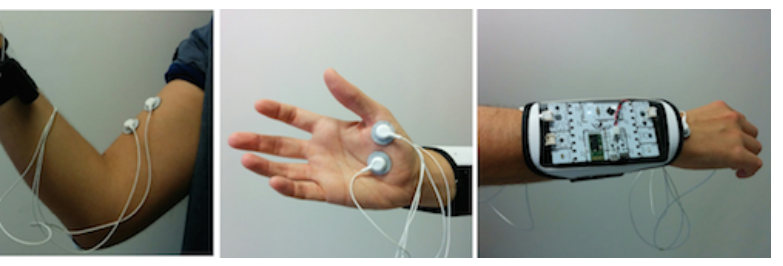

Fig. 6: Electrodes and sensor placement on the right arm. From left to right: EMG 1 (biceps), EMG 2 (thenar eminence), board placement on the wrist with incorporated ACC sensor. Note that the thenar eminence was used only for the evaluation reported in Section 4.1

the standard rehabilitation protocol and goals for post-stroke or post-operative recovery cases.

\subsubsection{Participants}

11 volunteers (4 males) were recruited from an association of retired people. The participants age ranged from 64 to 78 years $(73.2 \pm 4,4)$. Informed consent was obtained from all individual participants included in the study.

\subsubsection{Apparatus}

BITalino was used for the EMG and ACC data acquisition for both biofeedback methods. For the Method A a Rikomagic MK902II mini-PC with Android 4.4 was used, connected to a 14 inch monitor. While, for Method B, a Nexus 5 smartphone with Android 5.0.1 and the Bot'n Roll mobile robotic platform was used. All the Android applications were developed using the MobileBIT framework. The experiment was monitored by a member of the research team.

\subsubsection{Procedure}

First, demographic data was gathered by means of a short questionnaire. After that, the participants were helped to adjust the BITalino onto the right arm. Subsequently a calibration phase was carried out to find out the MVCs and define the activation threshold. The EMG electrodes were placed over the muscle fibres of the biceps, with a distance of approximately $2 \mathrm{~cm}$. The reference electrode was placed on a bone area on the elbow. The ACC sensor is on the BITalino board, which was placed on the right wrist of the user, as shown in Fig. 6. The sensor placement followed the recommendations established by the SENIAM task force [32].

In our experimental evaluation we considered an activation threshold of around $20 \%$ of the MVC, this threshold should be changed to a lower one if the participants shows fatigue while performing the experiment. Then, the users were required to perform a specific task. The task consists in the exercise routine chaining the exercises explained in
Table 3: The routine is composed of 3 different exercises in a sequence of 14 exercises

\begin{tabular}{lll}
\hline Type & Exercise description & Sequence order \\
\hline 1 & Biceps contraction & $1,3,5,7,8,10,12,14$ \\
2 & Turn wrist to the left & $2,4,6$ \\
3 & Turn wrist to the right & $9,11,13$ \\
\hline
\end{tabular}

Section 3.3.2. These exercises are chained and repeated in a routine of 14 movements, organized as shown in Table 3. Each of the exercises in the routine had to be maintained for $T=12$ seconds to be considered successful. Once an exercise is finished, the next one is performed until completing the whole routine.

The whole task is repeated twice for each method:

- In Method A, the participant had to follow the indications of the Android TV application to perform the task and complete the exercises. The screen showed a counter from 30 to 0 . Visual biofeedback was implemented in the following way: while the exercise was being performed well the counter counts down. If there was an error the counter resets to 30 . Whenever the countdown reached 0 the exercise was considered to be successfully performed and the next exercise was prompted on the screen.

- In Method B, the participants were provided with a sheet on which the routine was described and they had to perform the exercises watching the robot. When required, the researcher indicated the next movement to the participants. This method implements the animatronic biofeedback approach as described in Section 3.3.2.

During the experiment, the time required to complete the routine and the number of errors made were recorded. Once the participants had finished the evaluation, they were interviewed using two questionnaires: the System Usability Scale (SUS) [31] and the preferences questionnaire described in Table 4. The questionnaire was filled in by a research assistant to avoid misunderstandings of questions and Likert scales. After finishing the experiment, every participant was rewarded with a ticket for a meal in the Association restaurant.

\subsubsection{Design}

The experiment followed a within-subject design. Biofeedback method condition was counterbalanced using a Latin square. For the same routine two biofeedback methods were tested, visual biofeedback and animatronic biofeedback, and two attempts are performed to mitigate the learning effect. The routine was composed of three different types of exercises: 8 biceps contraction, 3 right wrist movements and 
Table 4: The second questionnaire is an 8 items Likert scale with 7 response options ( 1 totally disagree and 7 totally agree)

\begin{tabular}{lll}
\hline & Factor & Statement \\
\hline 1 & User Satisfaction: Lack of difficulty & I'm satisfied with the ease with which I completed the task. \\
2 & User Satisfaction: Perceived time to complete the task & I'm satisfied with the time I spent to complete the task. \\
3 & User Satisfaction: Comfort & I've felt comfortable using the system. \\
4 & User Satisfaction: Amusement & I've enjoyed using this system. \\
5 & User Awareness: Biceps movements & I was aware that the biceps contraction exercise was well performed. \\
6 & User Awareness: Wrist movements & I was aware that the wrist tilt exercise was well performed. \\
7 & Where the system is to be applied: Rehabilitation center & I would like to use this system in a rehabilitation center. \\
8 & Where the system is to be applied: Home & I would like to use this system at home.
\end{tabular}

3 left wrist movements. Aside from calibration, the number of expected entries were 11 participants $\times 2$ Methods $\times 2$ attempts $\times 14$ exercises/attempt $=616$ exercises $(352$ biceps, 132 left, 132 right). For each routine, a minimum time of 14 exercises/routine $\times 12$ seconds/exercise $=168$ seconds/routine are expected.

\subsection{Results and Discussion}

One of the volunteers did not finish the experiment because she could not maintain the required biceps contraction for $T=12$ seconds. Since that participant did not complete the routine, the corresponding questionnaires were not filled in. The rest of the participants (10) successfully completed the experiment.

Concerning the perceived usability of the system, the obtained SUS scores were $88.5 \% \pm 7.2$. Therefore, we conclude that participants considered our system to be usable. Regarding the required time to finish all the exercises and the error rate, we found that the results were similar for both methods (see Table 5 and Table 6).

With reference to the learning effect, in the second attempt, the time decreased by 11 seconds $(4 \%)$ for Method A and 25 seconds $(8 \%)$ for Method B. Conversely, the number of errors decreased by $5 \%$ for Method A but increased by $12 \%$ for Method B (see Fig. 7). The reduction in time by 25 seconds along with the slight increase by 2 in the number of errors, could suggest that the participants learnt how to deal quickly with the errors after the animatronic biofeedback during the second attempt.

To further compare both methods, we considered the second attempt as a repetition. Therefore, we increased the sample to $N=20$ values for each method. Afterwards, using the Shapiro-Wilk Normality Test we found that the data did not follow a normal distribution for Method A. Consequently we apply a Wilcoxon Signed-Rank test to analyse differences for the completion times and the number of errors between Method A and B. Regarding the time required to complete the routine, the medians of Method A and Method B were 249.93 and 291.56 respectively. The Wilcoxon Signed-rank test suggests that there is no signifi-
Table 5: Mean routine completion times in seconds

\begin{tabular}{lllll}
\hline Participant & \multicolumn{2}{c}{ Attempt 1 } & \multicolumn{2}{c}{ Attempt 2 } \\
& A (Visual) & B (Robot) & A (Visual) & B (Robot) \\
\hline 1 & 209.3 & 242 & 226.9 & 214.8 \\
2 & 230.1 & 3144 & 220.1 & 351.2 \\
3 & 235.7 & 258.8 & 230.1 & 228.4 \\
4 & 343.4 & 289.2 & 274.2 & 328.9 \\
5 & 319.7 & 300 & 338.5 & 250.4 \\
6 & 259.7 & 360 & 247.4 & 310.8 \\
7 & 373.8 & 241.6 & 279.3 & 294 \\
8 & 280.4 & 408 & 345.7 & 300.4 \\
9 & 252.5 & 239.2 & 212.5 & 232.8 \\
10 & 214.9 & 339.3 & 241.7 & 232.4 \\
\hline$\mu$ & 272 & 299.2 & 261.6 & 274.4 \\
$\sigma$ & 56.4 & 57.06 & 47.6 & 48.3 \\
\hline
\end{tabular}

Table 6: Number of errors registered in the experiment

\begin{tabular}{lllll}
\hline \multirow{2}{*}{ Participant } & \multicolumn{2}{c}{ Attempt 1 } & \multicolumn{2}{c}{ Attempt 2 } \\
& A (Visual) & B (Robot) & A (Visual) & B (Robot) \\
\hline 1 & 1 & 0 & 5 & 3 \\
2 & 7 & 12 & 2 & 28 \\
3 & 4 & 8 & 6 & 3 \\
4 & 26 & 7 & 14 & 23 \\
5 & 24 & 12 & 24 & 9 \\
6 & 9 & 17 & 7 & 15 \\
7 & 25 & 2 & 8 & 11 \\
8 & 8 & 31 & 28 & 20 \\
9 & 3 & 5 & 1 & 4 \\
10 & 1 & 8 & 8 & 4 \\
\hline$\mu$ & 10.8 & 10.2 & 10.3 & 12 \\
$\sigma$ & 10.2 & 8.8 & 9.1 & 9.1 \\
\hline
\end{tabular}

cant effect for method $(W=72, Z=-1232, p>0.05)$. With respect to the number of errors, the medians of Method A and Method B were 7.5 and 8.5 respectively. The Wilcoxon Signed-rank test shows that there is no significant effect for method $(W=94.5, Z=-0.392, p>0.05)$. These results, while not conclusive, do however, suggest that for this experiment the participants performance was identical in terms of completion time and errors, for both animatronic and visual feedback. 


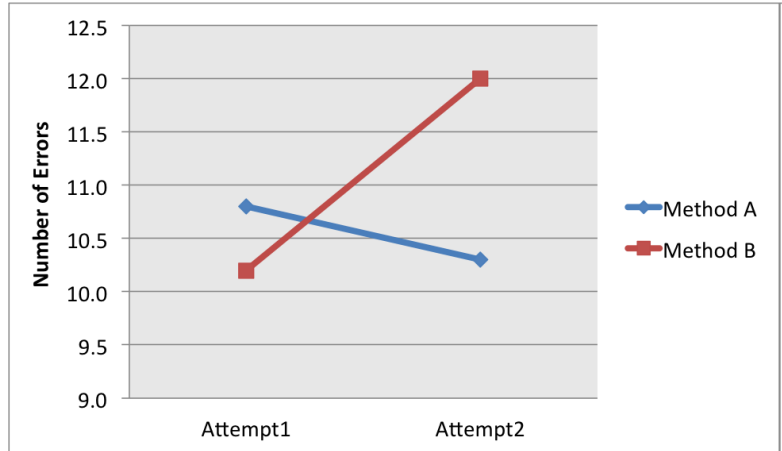

(a) Time to complete the routine

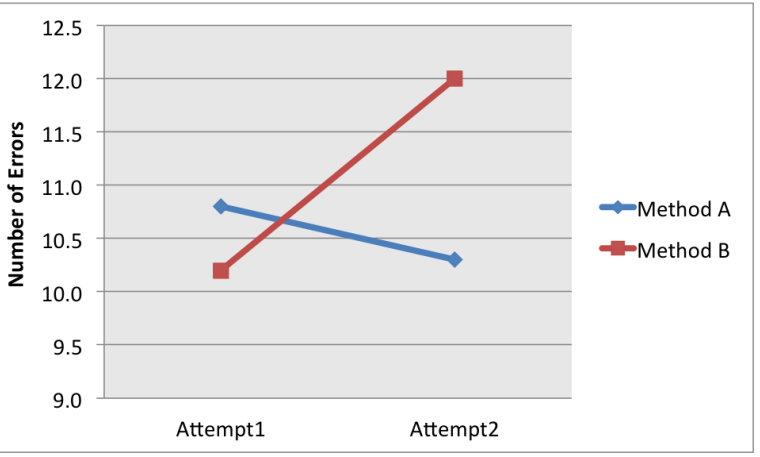

(b) Number of errors

Fig. 7: Progression charts for the two methods regarding the time and number of errors

Finally, the results of the interview and questionnaires show that both systems are perceived similarly (see Fig. 8). Nevertheless there is a noticeable difference for the Amusement item of the animatronic biofeedback. For this case, the medians of Method A and Method B were 5.5 and 7 respectively. A Wilcoxon Signed-rank test was applied to a sample of $N=7$ data (the test ruled out 3 participants for scoring both methods with the same value). Under these conditions, the Wilcoxon Signed-rank test shows that the method does have a significant effect ( $W=2$ and the critical value of $\mathrm{W}$ for $N=7$ at $p \leq 0.05$ is 2 ). This result is reinforced by some comments made by participants during the interview: "The robot is a toy", "It's funny". In addition, one participant also said that he felt more comfortable with the screen but that it was boring compared to the robot. As the main drawback, most of the participants believed that the visual feedback was more appropriate for home due to the space required for the movements of the robot. To overcome space restrictions another type of robots like robotic arms or humanoid robots should be tested.

\section{Conclusions}

In this paper we proposed and evaluated a context-aware framework, aimed at using animatronic biofeedback as a way of potentially increasing the compliance of elderly users to physical rehabilitation exercises performed at home. The results showed that animatronic biofeedback produced similar performance to visual biofeedback in completion time and error rate. The main benefit of this approach was the increase in the participants' satisfaction while doing the routines. This fact combined with some comments from the participants described in the previous section suggested that animatronic biofeedback can have a positive effect on the attention and engagement of the users. Further studies should be required to validate this finding.
Acknowledgements The authors gratefully acknowledge the support of the Spanish Ministry of Economy and Competitiveness through Project eGovernability (TIN2014-52665-C2-1-R). EGOKITUZ is funded by the Department of Education, Universities and Research of the Basque Government (grant IT395-10). The authors would also like to acknowledge the support of the Portuguese Fundação para a Ciência e Tecnologia (FCT) under the grant SFRH/BD/65248/2009, the company Bot'n Roll for support in use of their platform as well as the Jatorra retired people association located in Donostia for giving us the opportunity to make the experiment in their facilities. We also want to thanks Priscilla Alves, André Lourenço and Ana Fred for their collaboration in the foundational work for this research [30].

\section{References}

1. European Agency for Safety and Health at Work, Musculoskeletal disorders: Key facts. https://osha.europa.eu/en/topics/ msds/index_html/facts_html [accessed 14 July 2015].

2. UN World Health Organization (WHO), World Report on Disability. 2011, WHO/NMH/VIP/11.01, available at: http: //www . who.int/disabilities/world_report/2011/report.pdf [accessed 14 July 2015].

3. Giggins O.M., Persson U.M., Caulfield B., Biofeedback in rehabilitation. J Neuroeng Rehabil, 10(60), 2013.

4. Schwartz M. S., and Andrasik F., Biofeedback: A Practitioner's Guide. The Guilford Press, 2005.

5. Koh C.E., Young C.J., Young J.M., Solomon M.J., Systematic review of randomized controlled trials of the effectiveness of biofeedback for pelvic floor dysfunction. Br J Surg., 95(9):10791087, 2008.

6. Bo K., Berghmans B., Morkved S., Kampen M., Evidence-Based Physical Therapy for the Pelvic Floor: Bridging Science and Clinical Practice. Elsevier, 2007.

7. Coulter C.L., Scarvell J.M., Neeman T.M., Smith P.N., Physiotherapist-directed rehabilitation exercises in the outpatient or home setting improve strength, gait speed and cadence after elective total hip replacement: a systematic review. J Physiother, 59(4):219-226, 2013.

8. Rao S.S.C., Biofeedback therapy for constipation in adults. Best Pract Res Clin Gastroenterol, 25(1):159-166, 2011.

9. Petrofsky J.S., The use of electromyogram biofeedback to reduce Trendelenburg gait. Eur J Appl Physiol, 85(5):491-495, 2001.

10. Chandra, H., Oakley, I. and Silva, H., User needs in the performance of prescribed home exercise therapy. In Proc. of CHI '12, pages 2369-2374, 2012. 


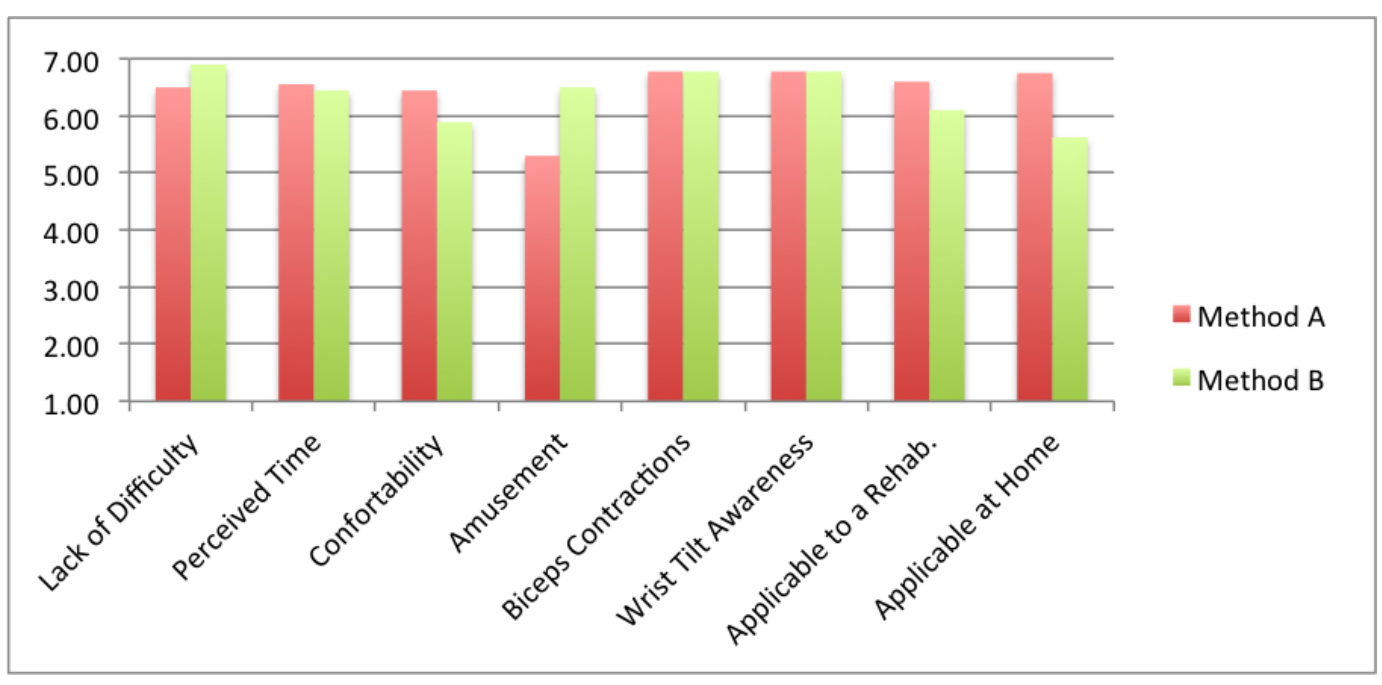

Fig. 8: Mean values obtained for the results of the second questionnaire (see Table 4)

11. Liu, Y. and Quian, G., Projector-Camera Guided Fast Environment Restoration of a Biofeedback System for Rehabilitation. In Proc. of the IEEE Conference on Computer Vision and Pattern Recognition, pages 1-2, 2007.

12. Lange, B., Chang, C.Y., Suma, E., Newman, B., Rizzo, A.S., Bolas, M., Development and evaluation of low cost game-based balance rehabilitation tool using the Microsoft Kinect sensor. In Proc. of the IEEE Eng Med Biol Soc Conf, pages 1831-1834, 2007.

13. Daponte, P., de Vito, L., Sementa, C., Validation of a home rehabilitation system for Range of Motion measurements of limb functions. In Proc. of IEEE Int. Symp. on Medical Measurement and Applications (MeMeA), pages 288-293, 2013.

14. Aung, Y.M., and Al-Jumaily, A., Shoulder rehabilitation with biofeedback simulation. In Proc. of the International Conference on Mechatronics and Automation, pages 974-979, 2012.

15. Farjadian, A.B., Sivak, M.L., Mavroidis, C., SQUID: Sensorized shirt with smartphone interface for exercise monitoring and home rehabilitation. In Proc. of the IEEE Int'l Conf. on Rehabilitation Robotics (ICORR), pages 1-6, 2013.

16. Daponte, P., de Vito, L., Pavic, B., and Silva, H., Case study on muscle activation analysis in post-stroke rehabilitation patients. In Proc. of IEEE Int. Symp. on Medical Measurement and Applications (MeMeA), pages 360-365, 2011.

17. Thought Technology Ltd, Comercial device list. http:// thoughttechnology.com/index.php/hardware.html [accessed 14 July 2015]

18. MindMedia Neuro and Biofeedback Systems, http://www. mindmedia.info/CMS2014/ [accessed 14 July 2015].

19. PLUX, Physioplux. http://www. physioplux.com/ [accessed 14 July 2015].

20. Sugar, T.G., He, J., Koeneman, E.J., Koeneman, J.B., Herman, R., Huang, H., Schultz, R.S., Herring, D.E., Wanberg, J., Balasubramanian, S., Swenson, P., and Ward, J.A., Design and control of RUPERT: a device for robotic upper extremity repetitive therapy. IEEE Trans Neural Syst Rehabil Eng, 15(3):336-46, 2007.

21. Lunenburger, L., Wellner, M., Banz, R., Colombo, G., Combining Immersive Virtual Environments with Robot-Aided Gait Training. In Proc. of the IEEE Conference on Rehabilitation Robotics, pages 421-424, 2007.

22. Monaco, V., Galardi, G., Coscia, M., and Martelli, D., Design and Evaluation of NEUROBike: A Neurorehabilitative Platform for Bedridden Post-Stroke Patients. IEEE Transactions on Neural Systems and Rehabilitation Engineering, 20(6):845-852, 2012.
23. Shull, P.B., Silder, A., Shultz, R., Dragoo, J.L., Besier, T.F., Delp, S.L., Cutkosky, M.R., Six-week gait retraining program reduces knee adduction moment, reduces pain, and improves function for individuals with medial compartment knee osteoarthritis. J Orthop Res., 31(7):1020-1025, 2013.

24. Casellato, C., Pedrocchi, A., Zorzi, G., Vernisse, L., Ferrigno, G., Nardocci, N., EMG-based visual-haptic biofeedback: a tool to improve motor control in children with primary dystonia. IEEE Trans Neural Syst Rehabil Eng., 21(3):474-480, 2013.

25. Banerji, S., Heng J., Ponvignesh, P.S., Menezes, D.D., Augmenting Rehabilitation after Stroke: A Flexible Platform for Combining Multi-channel Biofeedback with FES. Converging Clinical and Engineering Research on Neurorehabilitation Biosystems and Biorobotics, 259-263, 2013

26. Ribeiro A.F., Lopes G., Pereira N., Cruz J., Costa M.F.M., Bot'n roll robotic kit as a learning tool for youngsters. In Proc. of the 9th International Conference on Hands on Science (HSCI'2012), 192, 2012.

27. Silva H., Lourenço A., Fred A., Martins R., BIT: Biosignal igniter toolkit. Computer Methods and Programs in Biomedicine, 115(1):20-32, 2014

28. Dey A., Abowd G D., Salber D., A conceptual framework and a toolkit for supporting the rapid prototyping of context-aware applications. Hum.-Comput. Interact., 16(2):97-166, 2001.

29. Cânovas M., Silva H., Lourenço A., Canento F., Fred A., MobileBIT: A framework for mobile interaction recording and display. In Proc. of the 6th Conference on Health Informatics (HEALTHINF), SciTePress, 366-369, 2013.

30. Gamecho B., Guerreiro J., Alves A.P., Lourenço A., Silva H., Gardeazabal L., Abascal J., Fred A., Evaluation of a ContextAware Application for Mobile Robot Control Mediated by Physiological Data: The ToBITas Case Study. In Ubiquitous Computing and Ambient Intelligence. Personalisation and User Adapted Services, LNCS 8867, 147-154, 2014.

31. Brooke J., SUS: A quick and dirty usability scale. Usability evaluation in industry. Taylor and Francis, London, 1996.

32. SENIAM Project, Surface ElectroMyoGraphy for the NonInvasive Assessment of Muscles, available at: http://www . seniam.org/ [accessed 14 July 2015]. 